\title{
MAJOR ARTERIAL INJURY COMPLICATING FRACTURE OF THE FEMORAI. SHAFT
}

\author{
J. R. KirKuP, BATH, ENGLAND
}

A penetrating wound with damage to a major artery leads to immediate surgical exploration because the wound and bleeding draw attention to the pathology. Closed injuries causing arterial interruption (Couves, Lumpkin and Howard 1958) have often received less urgent consideration. On the supposition that the occlusion is due to arterial spasm, particularly in the presence of a fracture, treatment by reduction, sympathetic block and other conservative measures may be continued for too long before exploration is decided upon. If restoration of the circulation requires surgical intervention this must be completed within ten to twelve hours of injury at the most (Griffiths 1948, Murray 1952). Hardy and Tibbs (1960) showed that technically sound arterial reconstruction with restoration of distal pulsation is of no avail when performed beyond this critical time limit. Reminding us of the vast experience of arterial surgery now available, Birt (1961) also has made a plea for an aggressive attitude to interruptions of the femoral, popliteal and brachial arteries and has shown that there is little to lose and much to gain from re-establishing the arterial circulation as soon as the patient is resuscitated from the initial shock.

In the case of damage to the femoro-popliteal artery associated with fracture of the femoral shaft the treatment of shock and acute peripheral ischaemia is complicated by the problem of stabilising the fracture, especially when the break is near an arterial anastomosis. Published reference to this particular problem is scanty, perhaps because the injury is uncommon - though it may be seen more often if the volume and speed of road traffic continue to increase.

\section{CASE REPORTS}

Case 1-A man aged twenty-nine injured his left leg when he met with an accident while riding his motor-cycle. Examination on admission revealed a closed fracture of the left femoral shaft. The lowest third of the leg was cold and patchily blue, the ankle pulses were absent, and the foot was anaesthetic. The left thigh was moderately swollen and the patient was slightly shocked. Radiographs showed a transverse fracture just below the middle of the femoral shaft with slight shortening (Fig. 1).

A provisional diagnosis of arterial compression at the site of fracture was made and reduction by skeletal traction under radiographic control was performed. No circulatory improvement followed and a lumbar epidural block also failed to alter the situation. Meanwhile, the thigh did not swell abnormally, no bruit was heard and the patient's general condition remained good. A femoral arteriograph showed complete interruption of the artery at the level of the fracture. Some contrast medium was present in the popliteal artery (Fig. 2).

Operation-Operation was begun about eight hours after the injury. The left femoral artery and the lower two-thirds of the femur were approached through an antero-medial incision. In the adductor canal the artery was torn transversely and the ends were separated by an interval of about five centimetres; the adjacent vein was wounded opposite the fracture. Surprisingly little blood had escaped locally and no active bleeding from the artery or vein was seen. The fracture was reduced and the fragments were stabilised with a long plate. After limited mobilisation and freshening of the lacerated ends of the artery these were apposed with separate continuous sutures of the anterior and posterior walls. Heparin was instilled locally. The vein was ligated. 


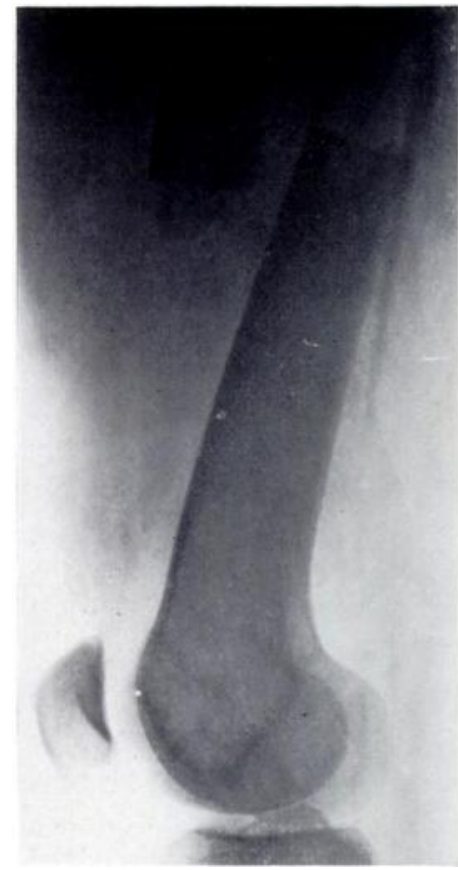

FIG. 1

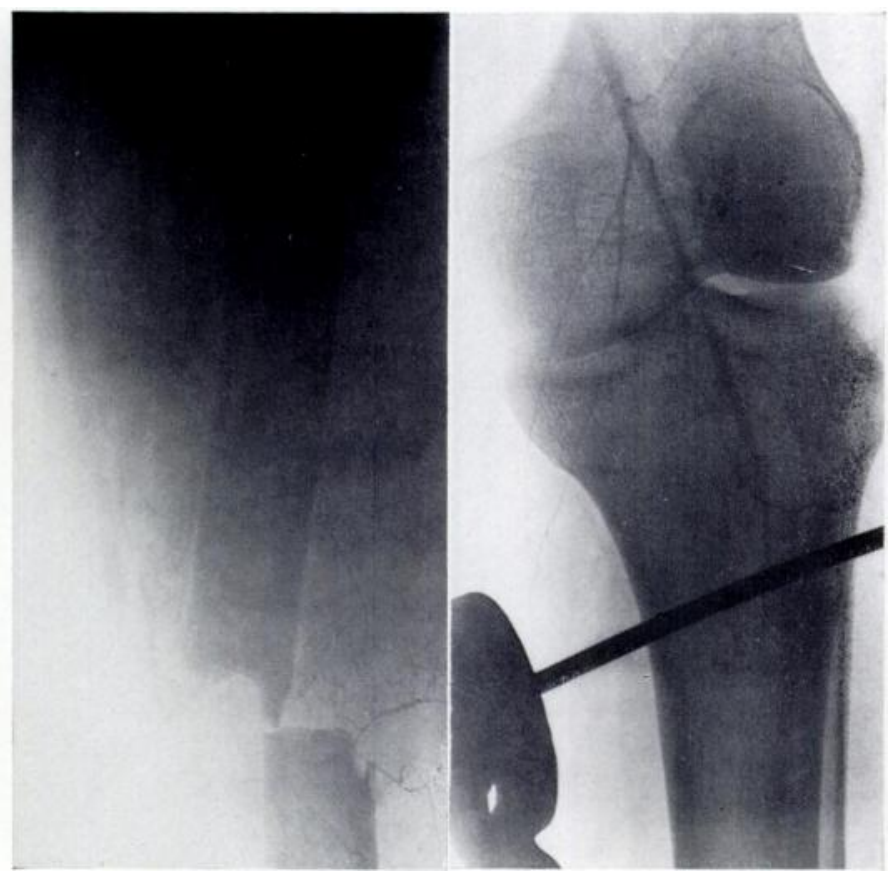

FIG. 2

Case 1. Figure 1-Lateral radiograph showing fracture through the midshaft of the femur. Figure 2Arteriographs done after reduction by skeletal traction. Note the interruption of the vessel at the level of the fracture and the relatively good filling of its distal part.

Progress-The distal pulses returned about ten hours after injury and remained palpable until the patient was lost to follow-up six months later. The leg, placed in a Thomas's splint and knee piece, was moderately oedematous for a week after the operation. At six weeks callus was evident on radiographs and the patient was got up in a hip spica. By six months he had regained almost full movement and had no vascular insufficiency. Post-operative arteriography was not performed.

Case 2-A man aged fifty-seven was thrown from a motorised truck and fell in front of the vehicle so that his right leg was trapped between it and a wall. On admission after being brought twenty miles by ambulance the patient was found to be shocked and to have a fracture of the right femur. There was a transverse wound five centimetres long across the right popliteal fossa, from which blood was oozing. The right foot was blue but, because of the low blood pressure, pulses were not felt in either foot. After transfusion, pulsation returned to the sound leg, but not to the right ankle and foot, which were found to have lost sensibility. Radiographs showed an oblique and comminuted fracture of the right femoral shaft just distal to the mid-point.

Operation-Operation began about seven hours after injury. Exploration by extension of the popliteal wound revealed a transverse division of the popliteal artery and vein in the upper part of the fossa, together with much blood clot and lacerated muscle. The bone end could be palpated through the upper part of the wound. The popliteal nerves were in continuity but dislocated from their tissue planes. The ends of the artery were four centimetres apart and were occluded by clot. The distal end of the vein was oozing. The ends of the vein were ligated and lacerated muscle was excised. The ends of the artery were freshened and approximated with interrupted everting sutures. Heparin was used locally. When the clamps were removed eight and a half hours after injury there was good distal filling of the artery. The wound was closed loosely and drained. The leg was immobilised with the knee well 
flexed on a Thomas's splint with a knee piece and seven pounds' skin traction was applied to the lower leg.

Progress-For several days there was general venous congestion of the leg, which became moderately oedematous. Peripheral pulsation was as good as that in the sound leg. On the fourteenth day a Steinmann pin was inserted through the uppermost part of the tibia in order to reduce the fracture, the knee being held flexed to avoid tension on the artery. Callus was shown on radiographs in the sixth week and by the eleventh week union was firm enough for the patient to be allowed to start walking without putting weight on the affected limb. Eleven months after injury the patient had a stable knee with a range of flexion from 180 to 100 degrees. The arterial flow remained satisfactory, though the distal pulses were less strong than those of the sound leg. Oedema gradually subsided, but unpleasant hyperaesthesia was experienced in the distal leg and foot when the patient first bore weight. This hyperaesthesia has persisted. An arteriograph done eleven months after injury showed normal flow in the main vessel with some narrowing at the site of anastomosis (Fig. 3). There was no evidence of circulatory insufficiency and the patient was able to walk half a mile without resting.

Case 3-A man aged twenty-one met with an accident while riding his motor-cycle. On admission after a sixteen-mile ambulance journey, the patient was almost pulseless and his blood pressure could not be recorded. There was a fracture of the right femur with marked swelling of the thigh, the right side of the abdomen and the right flank. Some grazes over the iliac crest were the only injuries of the skin. The right foot showed a patchy blue discoloration contrasting strongly with the general profound pallor. Saline was given intravenously and was followed by administration of two pints of plasma and five pints of blood. The general condition improved but the thigh continued to swell. All peripheral pulses except those at the right ankle returned with resuscitation. At that stage it was possible to detect that the right foot had lost sensibility and motor

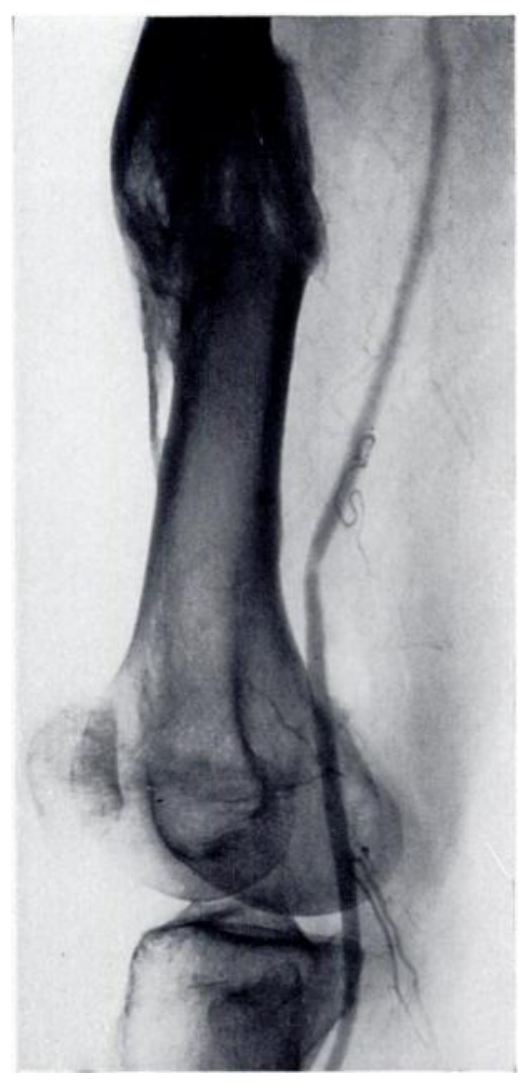

FIG. 3

Case 2-Arteriograph done elevein months after repair of popliteal artery. There is slight narrowing at the site of anastomosis; the fracture of the femur is united.

function. Radiographs showed a comminuted fracture of the femur just distal to the mid-shaft (Fig. 4). There was no evidence of pelvic fracture. A provisional diagnosis of injury to the femoral artery at the fracture site was made. The tenderness and swelling of the right flank raised the possibility of associated visceral injury.

Operation-Operation began eight and a half hours after injury. Exposure of the femoral vessels from just below the groin to the distal subsartorial canal was made through oedematous skin and gaping, tense, pale muscles. A large haematoma was removed and the femoral artery was exposed. It was in intense spasm above and below a lateral hole in its wall. There was a moderate amount of bleeding through this hole. The main vein was severed and the ends were occluded by clot. When the bleeding had been controlled the arterial laceration was seen to be adjacent to a large completely detached bone fragment. This was removed in order to avoid further damage to the vessels. The adventitia had been divided through about one-third of its circumference; there had been a transverse fracture of the intima and media,

VOL. 45 B. NO. 2, MAY 1963 
which had retracted inside the adventitia. After the adventitia had been divided and the ends of the vessel had been trimmed an end-to-end anastomosis with interrupted everting sutures was performed. Heparin was used locally. When the clamps were removed the arterial spasm relaxed. The ends of the vein were ligated and the skin was loosely closed. A Steinmann pin was inserted into the tibial tubercle and the leg was put on a Thomas's splint with a knee piece, the knee being kept well flexed. Five pounds traction was applied through the tibial pin. Because of the poor condition of the patient and the comminution of the femoral fragments, operative fixation of the fracture was not seriously considered. Restoration of full bone length would have rendered primary skin closure impossible because of the severe soft-tissue swelling. At the end of operation fifteen pints of fluid had been given intravenously.

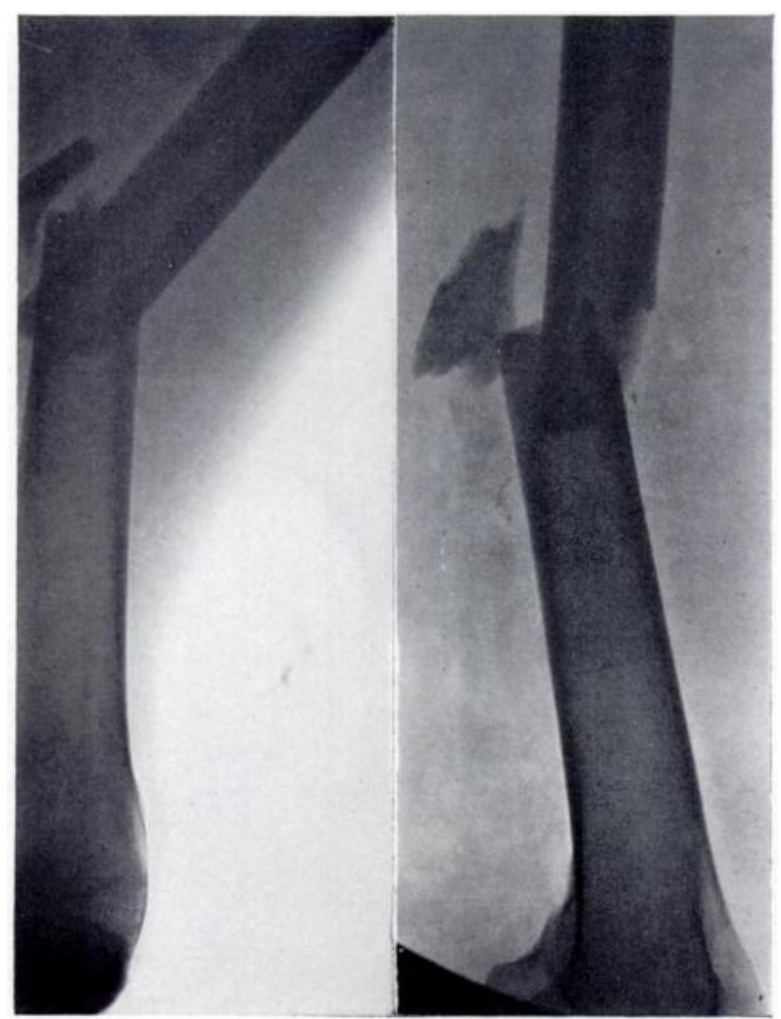

Fig. 4

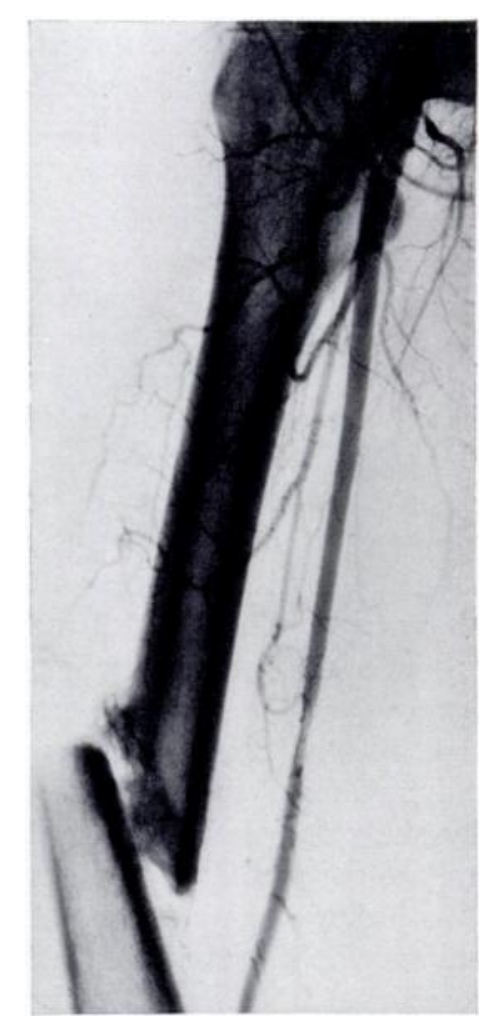

FIG. 5

Case 3. Figure 4-Antero-posterior and lateral radiographs showing fracture of femur. Note the large detached fragment. Figure 5-Arteriograph done four months after repair of femoral artery. There is slight narrowing at the site of suture.

Progress-The distal pulses returned ten hours after injury and were still palpable six months later. Soon after operation the whole limb became grossly oedematous. At this time the amount of urine produced was small; it was dark coloured and contained many red cells and much protein. On the sixth day there was a diuresis and after this the swelling of the leg began to subside. Overlap of the fragments was permitted so that there would be no tension at the arterial anastomosis. Union with shortening was thus unavoidable, but it was thought right to preserve the anastomosis and the limb and to encourage bony union even at the expense of some shortening and deformity. Union readily occurred and there was full recovery of sensory and motor function. Twelve weeks after injury the patient was up on crutches and within three months he went home wearing a caliper. A femoral arteriograph done four months after injury showed slight narrowing of the anastomosis with normal flow into the popliteal artery and beyond (Fig. 5). The limb was five centimetres shorter than the sound one. 


\section{DISCUSSION}

The incidence of arterial injury complicating fracture of the femoral shaft cannot be estimated from an experience of three cases at different hospitals. The few published accounts suggest that it is uncommon, but hospital records may be misleading because the injury itself or associated injuries may cause the patient's death in the ambulance or even on the road.

Details of one case are reported by Stein (1956) in a series of seven arterial injuries seen in orthopaedic practice. Reviewing 188 consecutive arterial injuries in a university department of surgery, Couves, Lumpkin and Howard (1958) found that thirteen patients had suffered closed damage to the limbs. Nine of these had suffered fractures or dislocations and one of these had an injury of the femoral artery associated with a femoral fracture. From the surgical department of another university Hardy and Tibbs (1960) report two more among eleven patients with traumatic ischaemia of the limbs. Three others are recorded by Birt (1961) in a study of nine major arterial interruptions seen during a five-year period at a large provincial hospital. One of these is reported as Case 1 in this series.

Causation-Road traffic accidents are often responsible for this complicated fracture. In this series two motor-cycles and one industrial vehicle initiated the violence. This was almost entirely spent on the injured thigh, because the associated injuries were minor ones.

Clinical picture-A fracture of the femoral shaft was associated with poor peripheral circulation characterised in each case by patchy cyanosis, coldness and anaesthesia of the foot and lower leg, loss of active movements of the toes and absence of the tibial pulses. The last four of these signs were not always immediately apparent, because of difficulty in assessment caused by severe shock. The patchy venous stasis is probably evidence of interruption of a large vein and points to major vascular injury in circumstances in which both vein and artery are liable to damage.

Loss of blood-Complete transverse division of a healthy major artery, as in Case 1, results in early natural arrest of bleeding and little blood is added to the fracture haematoma. This patient was only slightly shocked; swelling of the thigh was initially moderate and did not increase. Arterial compression in the fracture gap or local thrombosis due to contusion could have reproduced the same situation. The second patient also sustained transverse arterial division with early arrest of arterial bleeding, but was more shocked because of the open wound and the failure of clotting in the distal segment of vein. In contrast, when smooth retraction of the arterial ends is prevented, as with an oblique or incomplete division-as in Case 3-bleeding may continue to exsanguination. Such bleeding in the absence of a relieving wound produces a very large haematoma and the swelling may be sufficient to occlude the collaterals and so render the whole limb completely ischaemic. At operation in Case 3 the thigh was under great tension and the appearance of the muscles suggested such ischaemia. The oedema of the limb and the disturbance of renal function that occurred after operation may well have been caused by a reaction like that of the crush syndrome.

Diagnosis of arterial injury - Recognition of ischaemia calls for prompt and precise diagnosis of the arterial injury. A diagnosis of arterial spasm is only acceptable if compression by bone or fascial bands, laceration, rupture, subadventitial or subintimal haematomata or intimal fracture with thrombosis have been excluded (Collins and Jacobs 1961). In the three cases reported here the patients were seen soon after injury with clinical evidence suggestive of arterial interruption. Thus re-establishment of the circulation within the critical time limit was possible. In Cases 2 and 3 diagnosis was relatively easy, but in Case 1 the absence of a wound or massive haematoma made it more difficult. In such a case arteriography is justifiable if reduction of the fracture fails to produce improvement in the circulation. Demonstration of an undoubted block indicates the need for urgent exploration even if contrast medium is seen in the distal segment of the artery, because no reliance can be placed on the presence of

VOL. 45 B, NO. 2, MAY 1963 
back-flow (Hughes 1958). A blue, cold and insensitive foot is unlikely to become normal after conservative management even if gangrene does not supervene.

Mechanism of the fracture and arterial injury-This is often clear when the fracture is an open one. Direct violence applied to the femur injures the artery by trapping it against the bone or by driving bone fragments into the vessel. In Case 2 the vessels were divided by a projection trapping them against the back of the femur in the popliteal fossa. Probably the projection then slid up the shaft and broke the bone.

The mechanism in a closed injury is less clear. In Cases 1 and 3 both patients were motorcyclists who sustained fractures at the same level and had arterial lacerations opposite the fracture site in the middle of the femur. At this level the artery, lying in the subsartorial canal, is relatively mobile and is separated from the femur by muscle. In its progress from groin to popliteal fossa it takes a spiral course around the bone and in the middle part of the subsartorial canal is a medial or postero-medial relation of the femur. Only adduction displacement of the upper fragment or abduction with forward angulation of the lower fragment are likely to stretch the vessels over the bone. The initial radiographs in Case 3 show such displacement (Fig. 4). These features together with the findings at operation suggest that in spite of the protective muscular layer the upper fragment divided the artery in Case 1 and the lower fragment lacerated the artery in Case 3.

Treatment-Adequate resuscitation is necessary before operation. In Case 3 nine pints of fluid were given intravenously before operation and six more were given during operation to keep the blood pressure up. In desperate cases ligation may have to be accepted to preserve life, though at the femoro-popliteal level this will almost inevitably lead to gangrene or ischaemic change (Hughes 1958).

Management of the fracture-Fixation of the fragments by plating or intramedullary nailing eliminates any threat by the bone ends to the anastomosis. The circumstances in Case 1 created an ideal situation for internal fixation before arterial suture. In Case 3 both the general condition and the local situation made it impossible to contemplate internal fixation. However, the integrity of the anastomosis was preserved by keeping the knee well flexed and allowing the fragments to overlap. It is recognised that this course was by no means ideal. No information could be found about the time interval after which strong traction might safely be applied to a fracture adjacent to an arterial anastomosis.

The problem in Case 2 was rather different because the fracture site was remote from the site of vascular injury so that the anastomosis was not in danger of damage from the bone ends. Flexion of the knee to relieve tension on the anastomosis seemed the most important step, irrespective of stabilisation of the fracture.

In general, fixation of the fracture before arterial repair gives great advantages but there are circumstances in which it is better not to attempt it. It is not after all an essential step in achieving survival of the limb.

Technique of arterial repair-The technique required depends on the type of damage. Simple end-to-end suture was all that was necessary in these cases. Extensive lacerations or contusions with thrombosis call for excision and replacement by vein graft. Hughes (1958) has shown that the more complicated the procedure is, the less is the chance of successful restoration of circulation.

A lateral hole in a vessel following blunt injury must be regarded with suspicion because lateral suture only succeeds in stopping bleeding. Closer inspection may show that the intima and media are fractured completely and that the hole is in the adventitial sleeve. In such a case it is necessary to complete the division, remove clot from the distal segment, trim the ends and unite them by end-to-end suture.

Venous damage - Damage to the veins necessitated ligation in each case and led to temporary congestion and oedema mainly below the knee. One patient (Case 2) still has slight oedema eleven months after the accident. 


\section{SUMMARY}

1. Three cases of injury to the femoro-popliteal artery complicating fracture of the femoral shaft are described.

2. In all three cases restoration of peripheral circulation by arterial repair carried out within ten hours of injury succeeded in saving the limb from permanent damage.

3. The importance of adequate resuscitation, early diagnosis and early adequate surgical intervention is stressed.

4. The mechanism of injury and the clinical features of help in early diagnosis are discussed.

5. The place of internal fixation of the femoral fragments is discussed and its advantages and disadvantages are compared with those of treatment by skeletal or skin traction.

I wish to thank Mr J. G. Taylor and Mr A. B. Birt of Norwich and Mr V. S. Hughes-Davies and Mr A. E. Burton of Bath for their kind permission to publish these cases. I am also much indebted to $\mathrm{Mr}$ J. Bastow for his encouragement and advice.

\section{REFERENCES}

BiRT, A. B. (1961): The Treatment of Arterial Injuries in Civilian Life. In British Surgical Practice. Surgical Progress 1961. London: Butterworths.

Collins, H. A., and JACoBs, J. K. (1961): Acute Arterial Injuries Due to Blunt Trauma. Journal of Bone and Joint Surgery, 43-A, 193.

Couves, C. M., Lumpkin, M. B., and Howard, J. M. (1958): Arterial Injuries Due to Blunt (Non-Penetrating) Trauma: Experiences with Fifteen Patients. Canadian Journal of Surgery, 1, 197.

Griffiths, D. Ll. (1948): The Management of Acute Circulatory Failure in an Injured Limb. Journal of Bone and Joint Surgery, 30-B, 280.

Hardy, E. G., and TibBs, D. J. (1960): Acute Ischaemia in Limb Injuries. British Medical Journal, i, 1,001. Hughes, C. W. (1958): Arterial Repair During the Korean War. Annals of Surgery, 147, 555.

Murray, G. (1952): Surgical Repair of Injuries to Main Arteries. American Journal of Surgery, 83, 480.

Stein, A. H., Jun. (1956): Arterial Injury in Orthopaedic Surgery. Journal of Bone and Joint Surgery, 38-A, 669. 